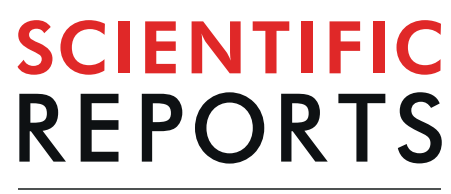

natureresearch

Check for updates

\title{
High-Throughput Generation of Bipod (Fab $\times$ scFv) Bispecific Antibodies Exploits Differential Chain Expression and Affinity Capture
}

\begin{abstract}
Thomas C. Nesspor, Kyle Kinealy, Nicholas Mazzanti, Michael D. Diem, Kevin Boye, Hunter Hoffman, Christine Springer, Justin Sprenkle, Gordon Powers, Haiyan Jiang, Sherry L. La Porte, Rajkumar Ganesan, Sanjaya Singh \& Adam Zwolak ${ }^{\bowtie}$
\end{abstract}

Generation of bispecific antibodies (BsAbs) having two unique Fab domains requires heterodimerization of the two heavy chains and pairing of each heavy chain with its cognate light chain. An alternative bispecific scaffold (Bipod) comprising an scFv and a Fab on a heterodimeric Fc eliminates the possibility of light chain mispairing. However, unpredictable levels of chain expression and scFv-induced aggregation can complicate purification and reduce the yield of desired Bipod. Here, we describe a highthroughput method for generation of Bipods based on protein $\mathrm{A}$ and $\mathrm{CH} 1$ domain affinity capture. This method exploits over-expression of the scFv chain to maximize heterodimer yield. Bipods purified by this method have purity suitable for cell-based functional assays and in vivo studies.

Bispecific antibodies (BsAbs) have generated significant interest for therapeutic development due to their novel mechanisms of action. BsAbs can be used for immune cell redirection, targeting multiple antigens or epitopes on a single antigen, immune checkpoint modulation, or to enhance the payload delivery of antibody-drug conjugates, with the number of applications ever expanding and nearly one hundred BsAbs in clinical development ${ }^{1-5}$.

Numerous BsAb formats have been developed ${ }^{6}$, and they can be grouped into those lacking an Fc region and those having an Fc region. Although each format has specific advantages, BsAbs harboring an Fc region are more prominent in research and clinical settings ${ }^{1}$ and are often favored due to their long serum half-lives mediated by FcRn-based recycling and due to the ability of the Fc region to mediate effector functions ${ }^{7}$. The Fc region can also be "silenced" to prevent effector function when desired ${ }^{8}$. BsAbs harboring an Fc region can be generated by either adding an additional binding moiety, such as a single-chain fragment variable (scFv) onto either the $\mathrm{N}$ - or C-terminus of either the heavy chain (HC) or the light chain (LC) to generate a symmetric BsAb. Alternatively, an asymmetric $\mathrm{BsAb}$ can be generated by introduction of mutations in the $\mathrm{HC} \mathrm{CH} 3$ domain, which forms most of the inter-chain contacts within the HC-HC interface, such that heterodimerization is favored over homodimerization. Asymmetric BsAbs are often advantageous over homodimeric BsAbs since they allow monovalent binding to each target. This is particularly important for $\mathrm{T}$ cell redirection approaches, since bivalent binding to $\mathrm{T}$ cells could lead to undesired activation and toxicity ${ }^{1}$.

Numerous sets of mutations that promote heterodimerization over homodimerization have been described ${ }^{9-11}$. In addition to $\mathrm{HC}$ heterodimerization, this approach necessitates a strategy to ensure proper pairing of the cognate light chains, and several solutions have been described. Introduction of complementary mutations in the HC-LC interface can drive proper pairing ${ }^{12-14}$. Other groups have used Fabs which share a common $\mathrm{LC}^{15}$, and still other groups have replaced one of the Fab arms with $\mathrm{scFv}$ or single domain $\mathrm{Abs}(\mathrm{VHH})$ to overcome the challenge of $\mathrm{HC}$-LC pairing ${ }^{16}$. Asymmetric BsAb formats lend themselves well to immune cell redirecting BsAbs such as the ones described here due to the preference for monovalent immune cell binding, and relatively close distance between the immune cell and cancer cell targeting arm, which drives effective immune synapse formation. 


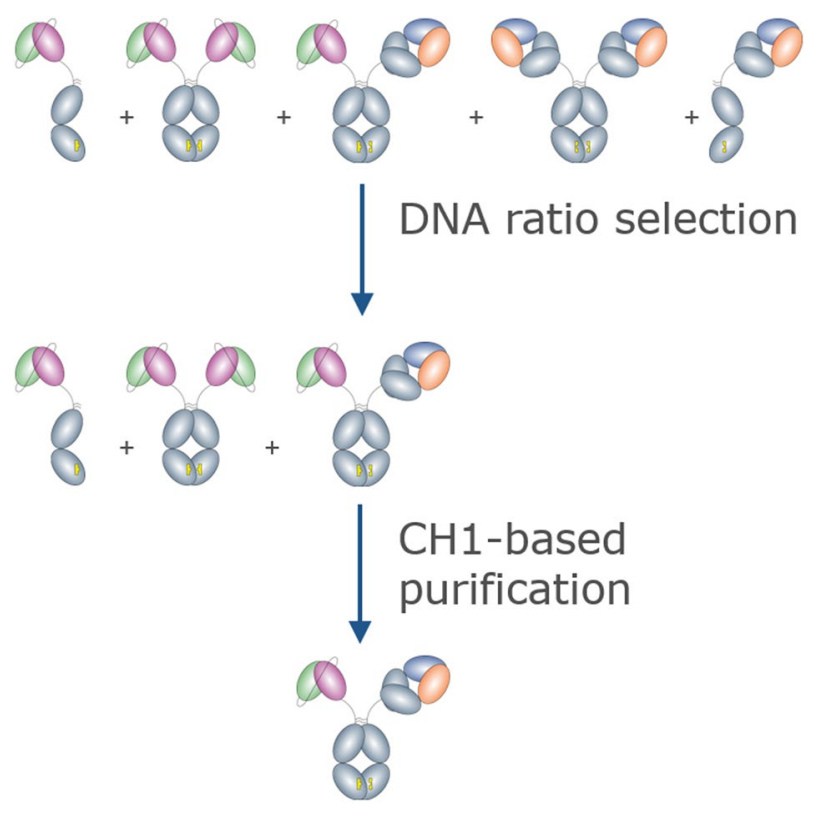

Figure 1. Cartoon illustration of the purification scheme which shows the potential species present after coexpression and purification of the "bipod" BsAb. Over-expression of the scFv-Fc arm saturates the HC-only species. Subsequent $\mathrm{CH} 1$-based purification selects only the desired heterodimeric BsAb.

Criteria for BsAbs suitable for clinical development include; relative ease of production, high stability, and favorable activity. To meet these criteria, many variables must be screened in a research setting, and therefore, methods to efficiently produce and screen large panels of high purity BsAbs are crucial. This is especially true for immune cell engaging BsAbs, as small amounts of contaminating homodimer can confound functional analysis. Here, we focus on a BsAb format, which we term a "Bipod", in which one of the binding arms is a Fab while the other is a scFv. To generate these asymmetric BsAb, one HC contains T350V, L351Y, F405A, Y407V mutations and the other HC contains the complementary T350V, T366L, K392L, T394W mutations which have previously been shown to enhance heterodimerization ${ }^{11,17}$. We describe a novel method for high-throughput purification of bipods, with purity suitable for downstream functional assays.

\section{Results}

DNA transfection ratio. We sought to identify a BsAb format and a method for generating BsAbs that would be suitable for high-throughput production of large panels, and which would result in highly pure molecules for functional and biophysical screening. We chose to use an asymmetric bispecific antibody comprising a full heavy chain paired with its cognate light chain on one subunit and an scFv fused to the Fc on the other subunit, since it eliminates the challenge of pairing two unique light chains with their appropriate heavy chains (Fig. 1). Each chain was expressed from its own plasmid having an identical promoter. The scFv-Fc and heavy chains featured complementary mutations designed to enhance heterodimerization described previously ${ }^{11}$. Briefly, the scFv-Fc chain (chain A) contained mutation of T350V, L351Y, F405A, Y407V and the heavy chain (chain B) contained mutation of T350V, T366L, K392L, T394W. Given equal expression of each heavy chain, these mutations in human IgG1 were shown to result in $\sim 95 \%$ heterodimeric species having biophysical properties similar to a wild-type IgG $1^{11}$. Expressed on its own, chain A exists as a population of $~ 90 \%$ half-Ab and $10 \%$ homodimer, whereas chain B is $\sim 40 \%$ half- $\mathrm{Ab}$ and $\sim 60 \%$ homodimer (Supplementary Fig. S1).

Light chain, which is required for heavy chain secretion ${ }^{18}$ was always over-expressed with respect to its cognate heavy chain. The scFv-Fc chain, heavy chain, and light chains were expressed using a DNA molar ratio of $2: 1: 3$. This DNA ratio resulted in over-expression of the scFv-Fc chain in $>95 \%$ of BsAbs tested. The excess scFv-Fc chain reduces the likelihood of unpaired Fab heavy chain or Fab-Fab heavy chain homodimer such that contaminants are limited to scFv-Fc only species (Supplementary Fig. S1).

$\mathrm{CH} 1$-based purification of BsAbs. Culture supernatants from cells transfected using non-equimolar plasmid ratios contained a limited number of species: target BsAb, scFv-Fc monomer and homodimer, and LC homodimers. To purify the target BsAb we selected an IgG-CH1 affinity resin, because due to the lack of Fab-Fab homodimer, BsAb was the only species in the supernatant containing a $\mathrm{CH} 1$ domain. We first sought to identify a DNA ratio of chain A to chain B which would be sufficient to over-express the scFv-Fc chain in a majority of BsAbs. Since the expression level of each polypeptide is related to the sequence of the variable region and can vary by several fold, we tested whether DNA ratios of 1:1:3 or 2:1:3 scFv-Fc:HC:LC would result in excess scFv-Fc for most Abs using the same method (Table 1). For a panel of ten BsAbs, the main peak, consisting of target BsAb and scFv-Fc was $\sim 67 \%$ for a DNA ratio of $1: 1: 3$ compared to $81 \%$ for a DNA ratio of $2: 1: 3$, suggesting that two-fold over-expression of the $\mathrm{scFv}$-Fc chain would be generally sufficient to limit off-target species. 


\begin{tabular}{|l|l|l|}
\hline \multirow{2}{*}{ Sample } & \%Main peak \\
\cline { 2 - 3 } & $\mathbf{1} 1: \mathbf{1}$ & $\mathbf{2}$ \\
\hline BsAb70 & 81 & 84 \\
\hline BsAb71 & 71 & 80 \\
\hline BsAb72 & 65 & 86 \\
\hline BsAb74 & 65 & 90 \\
\hline BsAb75 & 81 & 75 \\
\hline BsAb76 & 63 & 85 \\
\hline BsAb77 & 50 & 73 \\
\hline BsAb78 & 77 & 85 \\
\hline BsAb79 & 69 & 77 \\
\hline BsAb80 & 43 & 77 \\
\hline
\end{tabular}

Table 1. Comparison of the \% Main SEC peak at two different DNA ratios.

A

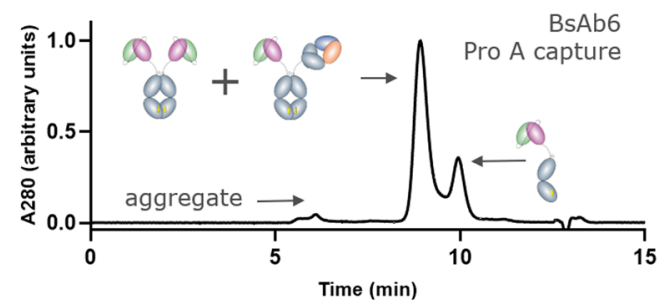

B

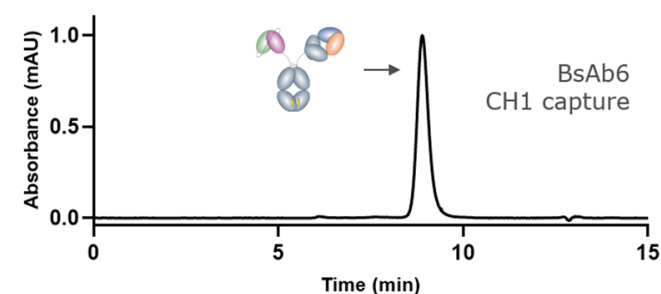

C



119

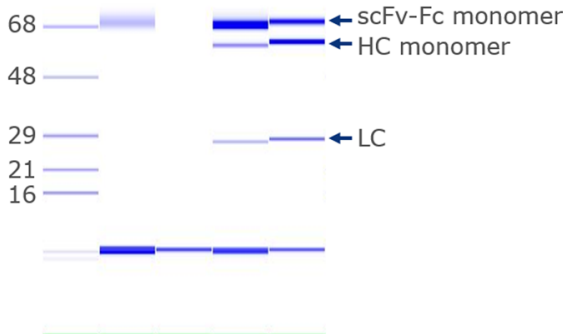

Figure 2. Example of a BsAb purified using the DNA imbalance $+\mathrm{CH} 1$ purification method. (A) Analytical SEC chromatogram of the initial protein A eluate featuring a main peak comprised of target heterodimer and scFv-Fc homodimer in addition to a minor peak comprised of scFv-Fc half Ab. (B) Analytical SEC chromatogram of the $\mathrm{CH} 1$ eluate from (A) showing capture of only the desired heterodimeric BsAb. (C) Non-reduced GXII analysis of the protein A eluate (lane 1) and the CH1 eluate (lane 2). Captured species are indicated on the gel image.

While IgG-CH1 capture could isolate the BsAb in a single step, we then sought to characterize the population of scFv-only species and target BsAb. Therefore, we first captured Fc-containing species using protein A affinity chromatography and analyzed the product using analytical size-exclusion chromatography (SEC) and capillary electrophoresis (Fig. 2, Table 2). The SEC chromatogram displayed 3 distinct peaks (Fig. 2A). A minor peak eluting at $\sim 6 \mathrm{~min}$ contained aggregated species. The major peak, eluting at $\sim 9$ min contained the target $\mathrm{BsAb}$ and scFv-Fc-only homodimer, as confirmed by capillary electrophoresis (Fig. 2C). Another peak eluting at $\sim 10$ min contained scFv-Fc monomer. For this panel, the estimated \% main peak ranged from 57 to $90 \%$ target species, based on quantitation of SEC (Fig. 2). The protein A eluate was then purified using CH1-affinity resin to remove species that lack a $\mathrm{CH} 1$ domain, or scFv-Fc only species, consistent with the capillary electrophoresis result (Fig. 2B,C). The resulting protein solutions were typically $>97 \%$ target BsAb. One antibody, BsAb2, was only $91 \%$ pure after $\mathrm{CH} 1$ capture, and this may have been due to instability of the scFv moiety, which caused time-dependent aggregation with Fab-containing species. To confirm that the $\mathrm{CH} 1$ capture method could be used for different panels of BsAbs, we showed that another panel, purified in the identical process, produced similar levels of protein purity even at target species levels as low as $21 \%$ after protein A purification (Supplementary Table S1).

We then asked whether "bipod" format BsAbs could be purified in one step using CH1 capture directly from culture supernatants. Five BsAbs were expressed using a 2:1:3 ratio of scFv-Fc:HC:LC and captured directly by $\mathrm{CH} 1$ affinity, and the product was analyzed by SEC (Fig. 3, Table 3). Because we have shown that $\mathrm{CH} 1$ affinity capture effectively eliminates scFv homodimer, we use main peak area as a surrogate for BsAb purity. The \% purity of each BsAb ranged from 88 to $96 \%$ BsAb. The slightly lower $\%$ target species for BsAbs produced by direct 


\begin{tabular}{|l|l|l|}
\hline Sample & $\begin{array}{l}\text { Estimated \% Main peak } \\
\text { post protein A }\end{array}$ & $\begin{array}{l}\text { Estimated \% Main peak } \\
\text { post CH1 capture }\end{array}$ \\
\hline BsAb2 & 57.1 & 91.5 \\
\hline BsAb3 & 83.3 & 99.1 \\
\hline BsAb4 & 84.5 & 99.3 \\
\hline BsAb5 & 80.8 & 99.3 \\
\hline BsAb6 & 70.9 & 99.3 \\
\hline BsAb7 & 80.3 & 97.4 \\
\hline BsAb8 & 84.9 & 99.5 \\
\hline BsAb9 & 77.8 & 98.9 \\
\hline BsAb10 & 89.7 & 99.4 \\
\hline BsAb11 & 82.8 & 98.3 \\
\hline
\end{tabular}

Table 2. Purity of BsAbs after protein A capture and a second $\mathrm{CH} 1$ affinity capture step.

\begin{tabular}{|l|l|}
\hline Sample & \% BsAb \\
\hline BsAb30 & 95.4 \\
\hline BsAb44 & 88.4 \\
\hline BsAb46 & 95.5 \\
\hline BsAb82 & 91.0 \\
\hline BsAb380 & 93.0 \\
\hline
\end{tabular}

Table 3. Analysis of BsAb purity after one-step $\mathrm{CH} 1$ purification.
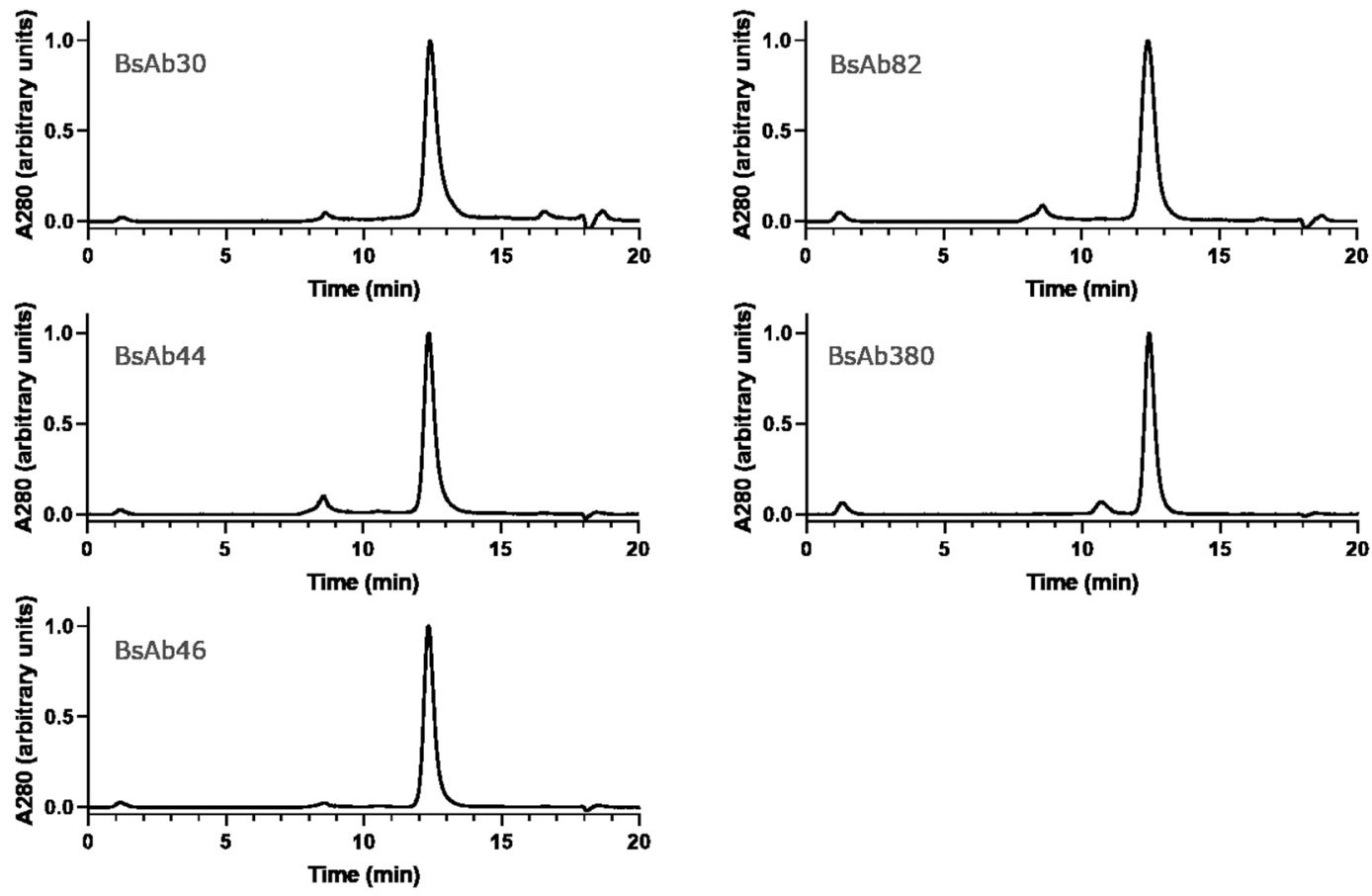

Figure 3. Examples of BsAbs purified using the direct, one-step $\mathrm{CH} 1$ purification method. Analytical SEC chromatograms of the $\mathrm{CH} 1$ eluate featuring a main peak comprised of target heterodimer and minor aggregate species.

$\mathrm{CH} 1$-based capture was generally due to aggregated protein, rather than excess homodimer or half-antibody. In general, BsAbs could be purified in one-step directly by $\mathrm{CH} 1$ capture yielding BsAbs $>90 \%$ pure.

HTP production of BsAbs using non-equimolar plasmid ratios. Since the $\mathrm{CH} 1$-based affinity purification of a small panel of BsAbs resulted in highly pure product, we then asked whether this method could be applied to larger panels of Abs produced at smaller scale in a high-throughput process. For this, we used a panel of $119 \mathrm{BsAbs}$ having either the $\mathrm{HC} \times \mathrm{scFv}-\mathrm{Fc}, \mathrm{scFv}-\mathrm{Fc} \times \mathrm{HC}$, or scFv-Fc $\times$ scFv-Fc (Fig. 4A) format. The 
A
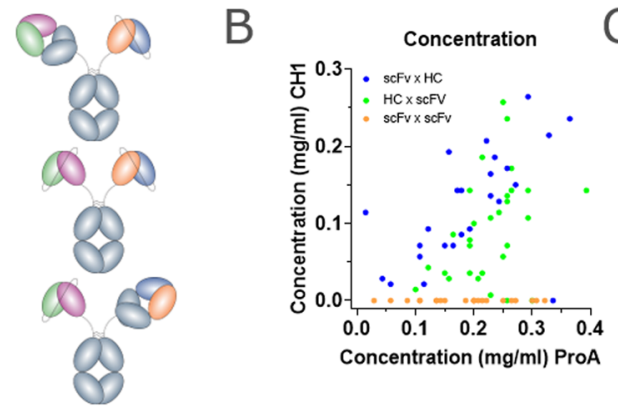

$\mathrm{E}$
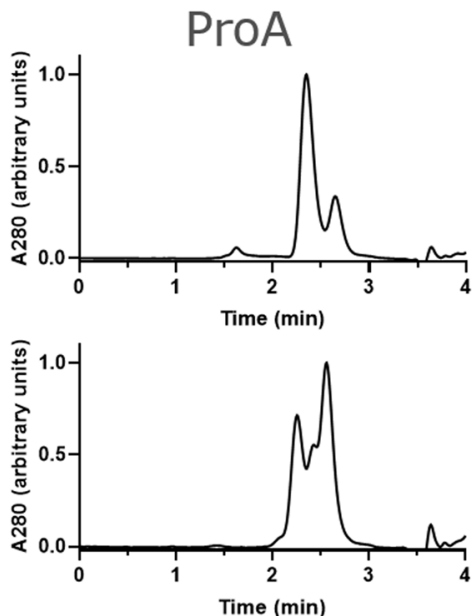

F
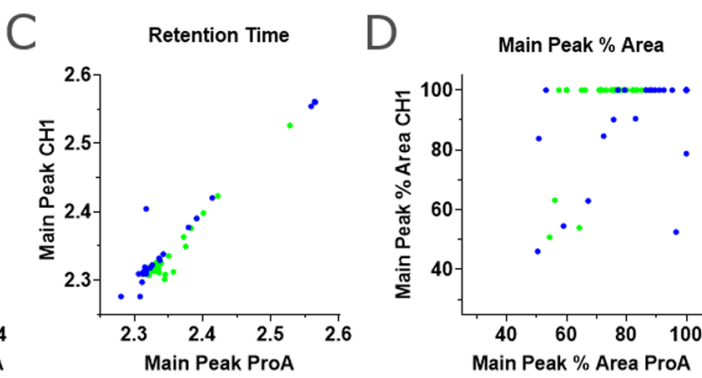

Figure 4. Analysis of BsAbs generated by DNA imbalance and $\mathrm{CH} 1$ capture using high-throughput methods. (A) Comparison of the total yield of antibody from $\mathrm{CH} 1$ capture vs protein A capture showing that over-expression of the $\mathrm{scFv}-\mathrm{Fc}$ arm generally results in saturation of the $\mathrm{HC}$ arm, allowing $\mathrm{CH} 1$ capture of highly pure BsAb. The two "bipod" format BsAbs $(\mathrm{HC} \times \mathrm{scFv}-\mathrm{Fc}$ or scFv $\times \mathrm{HC})$ are indicated in blue and green. scFv-Fc $\times$ scFv-Fc format bipods are shown in orange. (B) Comparison of the elution time of the main peak from $\mathrm{CH} 1$ capture vs protein A capture showing that the main peak is generally represented by target heterodimer and is consistent between methods. (C) Comparison of the \% of the total protein population represented by the main peak from $\mathrm{CH} 1$ capture vs protein A capture showing that $\mathrm{CH} 1$ capture generally results in highly pure target BsAb. (D,E) Analytical SEC analysis of two proteins which illustrate the requirement for over-expression of the scFv-Fc arm. In D, excess DNA of the scFv-Fc arm results in overexpression of this component, leading to saturation of the HC-only species during expression. After protein A capture, $\mathrm{CH} 1$ capture retains only target $\mathrm{BsAb}$. In E, over-expression of the $\mathrm{HC}$ component prevents its saturation with $\mathrm{scFv}-\mathrm{Fc}$ arm, and prevents isolation of the target BsAb after protein A capture.

BsAbs in the panel were purified either by protein $\mathrm{A}$ or by $\mathrm{CH} 1$-capture, and the concentration of the eluate was measured for each method (Fig. 4B). As expected, a set of scFv-Fc $\times$ scFv-Fc control BsAbs did not bind the $\mathrm{CH} 1$ resin, and thus resulted in no captured protein. For the set of "bipod" molecules, protein A capture yielded higher quantities of protein since it captures all $\mathrm{Fc}$-containing species, whereas $\mathrm{CH} 1$ capture yields were lower, ranging from $\sim 0.1 \mathrm{mg} / \mathrm{mL}$ to $0.3 \mathrm{mg} / \mathrm{mL}$ in $400 \mathrm{uL}$ with an average of $0.15 \mathrm{mg} / \mathrm{mL}$. To determine the composition of the captured protein, we analyzed each protein by SEC. The major peak eluted at the same retention time with either resin (Fig. 4C). To determine whether $\mathrm{CH} 1$ capture resulted in higher purity, we then determined the percent of the total population represented by the major peak, which for Protein A could contain both scFv homodimer and $\mathrm{BsAb}$, but for $\mathrm{CH} 1$ capture contains only BsAb. Indeed, $\mathrm{CH} 1$ capture resulted in a larger percentage of target $\mathrm{BsAb}$, as represented by the main peak percent area. This increase in purity would have been more pronounced had we been able to quantitate the amount of bsAb in the protein A-captured protein. Moreover, in 44 of 59 "bipod" proteins, CH1 capture resulted in $~ 100 \%$ target BsAb (Fig. 4D). Note that the "bipod" panel contained both $\mathrm{HC} \times \mathrm{scFv}-\mathrm{Fc}(\mathrm{scFv}$ chain was constant) and $\mathrm{scFv}-\mathrm{Fc} \times \mathrm{HC}$ (HC chain was constant) BsAbs. The $\mathrm{CH} 1$-based enrichment of target species for the $\mathrm{HC} \times \mathrm{scFv}-\mathrm{Fc}$ format occurred in 29 of 34 proteins, whereas the enrichment for scFv-Fc $\times$ HC format occurred in only 14 of 25 proteins. This was likely due to the lower expression level, on average, of the panel of variable $s c F v$ chains in this format and could be overcome by further increasing the ratio of $s c F v-F c$. These results suggest that if the $s c F v-F c$ chain is overexpressed, it would saturate all the $\mathrm{HC}$ and that $\mathrm{CH} 1$-based affinity capture could then selectively capture the target BsAb, whereas if the HC is over-expressed, the $\mathrm{CH} 1$-based capture will not result in highly pure BsAb. An example of each case highlights that over-expression of the scFv-Fc chain does in fact result in saturation of the HC, preventing HC homodimers and half-Abs (Fig. 4E,F). 
A

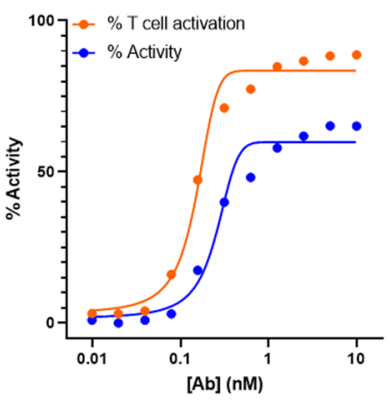

B

$$
\mathrm{EC}_{50}=32 \mathrm{pM}
$$

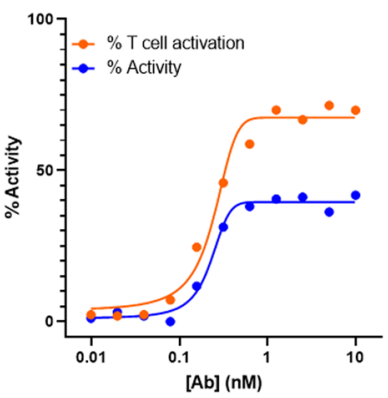

Figure 5. Functional comparison of a "bipod" BsAb produced using either large-scale (A) or high-throughput (B) methods. (A) Graph of the \% CARNAVAL cell lysis vs concentration of antibody overlaid with the relative $\mathrm{T}$ cell activation, based on CD25 expression. The BsAb displays an $\mathrm{EC}_{50}$ for CARNAVAL cell killing of $32 \mathrm{pM}$. (B) The same experiment in (A) was performed using the BsAb generated in small scale using high-throughput methods, and it shows the antibody has similar activity, displaying an $\mathrm{EC}_{50}$ for CARNAVAL cell killing of 23 pM.

BsAbs display anti-tumor cytotoxicity. Cell-based cytotoxicity assays require highly pure protein to generate reliable results. The need for consistently high purity is especially true when screening large panels of bsAbs, where activity differences may be minor. We asked whether BsAbs generated using our HTP method would have comparable activity to the same BsAbs made at large scale. For this, we measured T cell-based cytotoxicity against CARNAVAL B cell lymphoma cells (Fig. 5A,B, Supplementary Fig. S3). The BsAb showed similar $\mathrm{EC}_{50}$ for target cell killing $(0.032 \mathrm{nM}$ vs $0.023 \mathrm{nM})$ when produced at either scale. The maximum cytotoxicity values were slightly different, and this was due largely to variability in cell cultures, since $\mathrm{EC}_{50}$ values were similar across BsAbs produced in either large scale or HTP scale.

To illustrate how functional characterization of high purity BsAbs produced using our HTP process can be used for lead selection, a panel of 59 BsAbs was generated. This panel used three different anti-CD3 v-regions to redirect T cells: a high affinity $\left(\mathrm{K}_{\mathrm{d}} \sim 5 \mathrm{nM}\right)$, medium affinity $(\mathrm{Kd} \sim 20 \mathrm{nM})$, and a low affinity $(\mathrm{Kd} \sim 300 \mathrm{nM})$. The redirection arms were paired with tumor-targeting v-regions with $\mathrm{K}_{\mathrm{d}}$ ranging from $10 \mathrm{pM}$ to $3 \mathrm{nM}$. These were formatted either as $\mathrm{Fab} \times \mathrm{scFv}-\mathrm{Fc}$ or $\mathrm{scFv}-\mathrm{Fc} \times \mathrm{Fab}$ Abs. The panel showed a range of activities, from $>90 \%$ cytotoxicity to no activity (Fig. 6A). The cytotoxic activity of the BsAbs was highly dependent on affinity for CD3, affecting both the maximum cytotoxicity level and $\mathrm{EC}_{50}$ for killing (Fig. 6A, rows). Interestingly, modulation of the affinity of the targeting arm (from $\mathrm{K}_{\mathrm{d}}=50 \mathrm{pM}$ to $\mathrm{K}_{\mathrm{d}}=2.9 \mathrm{nM}$ ) affected $\mathrm{EC}_{50}$ but had less effect on maximum cytotoxicity (Fig. 6A, columns).

In another example of how our process can enable early functional characterization of large BsAb panels, we generated a panel of BsAbs to evaluate the effects of epitope on T cell activation. For this panel of bipod BsAbs, tumor lysis and $\mathrm{T}$ cell activation, measured by CD25 expression, were inversely correlated with the $\mathrm{EC}_{50}$ for target cell binding (Fig. 6B). T cell activation was directly correlated to target cell lysis (Fig. 6B). The tumor antigen targeted by this panel was comprised of six domains (D1-D6), with D1 being membrane distal and D6 being membrane proximal. Most BsAbs bound to the central D2-D4 domains (Table 4), and analysis of the large number of binders allowed interrogation of whether targeting certain domains would result in more favorable activity. BsAbs that bound the centrally located D3 domain on the target cell antigen displayed a range of activities, but on average showed higher target cell lysis, and average cytotoxicity $\mathrm{EC}_{50}$ of than those that bound the adjacent D2 and D4 domains (Fig. 6B, Table 4), suggesting that D3-binding BsAbs may be more desirable than those that bound D4. Overall, T cell activation, based on CD25 expression, appeared to be correlated with maximum and $\mathrm{EC}_{50}$ for cytotoxicity (Fig. 6B). This example clearly illustrates how screening of multiple variables for a large panel of BsAbs, enabled by our HTP process, provided critical data for the early selection of lead candidates.

BsAbs that redirect $T$ cells against target cells are often associated with high toxicity in clinical studies, and this toxicity is thought to be related to high relative affinity for T cells over target cells. Desirable T cell-redirecting BsAbs would display high cytotoxicity coupled with low T cell activation. Alternatively, some BsAbs may mediate target cell killing in the absence of $\mathrm{T}$ cell activation or effector function, such as those that can block signaling; for example, anti-EGFR Abs ${ }^{19}$. Thus, we measured activation of T cells, using CD25 expression as a confirmation of 
A

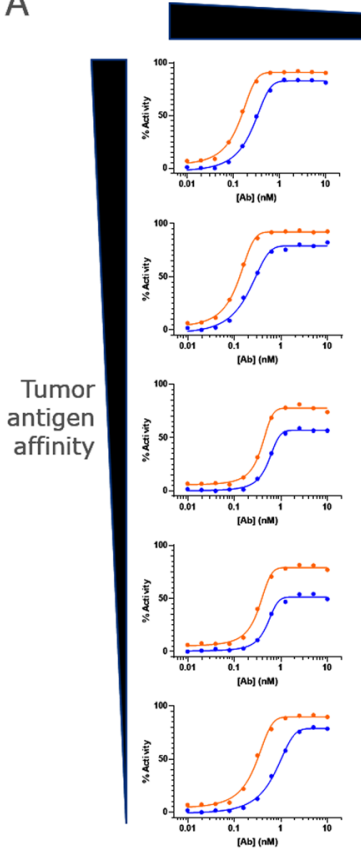

CD3 affinity
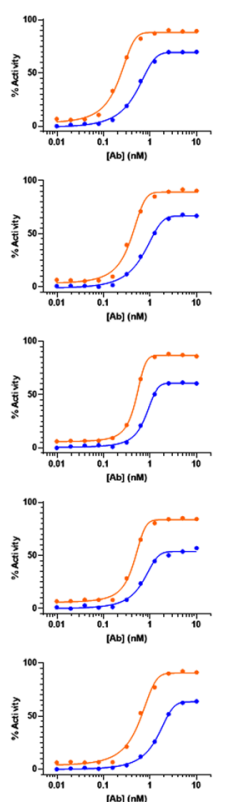
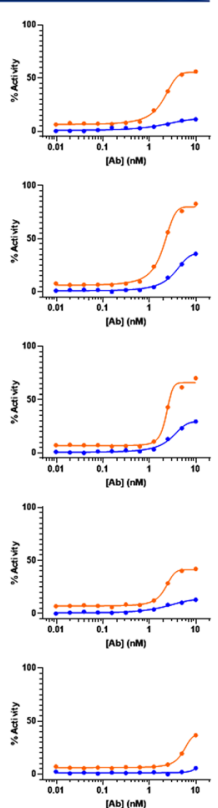

B
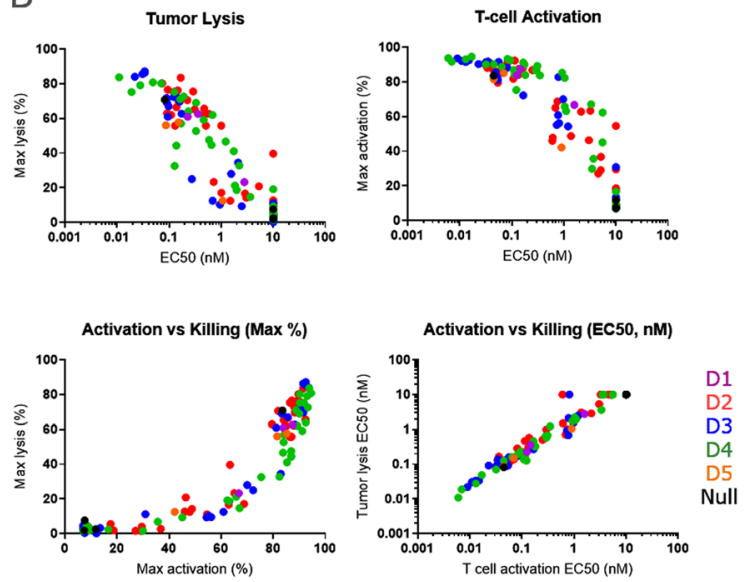

Figure 6. High-throughput generation of highly pure BsAbs allows interrogation of larger panels of BsAbs and analysis of wider functional questions. (A) Examples of CARNAVAL cell killing and T cell activation analysis using the same experiments as in (Fig. 4). BsAbs displayed a range of activities, from almost total cell killing, to total lack of activity. (B) The activities of a panel of BsAbs was analyzed and correlated with antigen binding epitope. Generation of large panels of highly pure, functional BsAbs allows interrogation of a range of antigenbinding affinities, epitopes, and format which generally present a technical challenge of purifying BsAbs since small levels of impurity can lead to large changes in activity.

\begin{tabular}{|l|l|l|l|l|l|l|}
\hline $\begin{array}{l}\text { Binding } \\
\text { Domain }\end{array}$ & $\begin{array}{l}\text { No. } \\
\text { Abs }\end{array}$ & $\begin{array}{l}\text { Avg. Kd } \\
(\mathbf{n M})\end{array}$ & $\begin{array}{l}\text { Average Cytotoxicity } \\
(\text { EC50, } \mathbf{n M})\end{array}$ & $\begin{array}{l}\text { Average max. } \\
\text { cytotoxicity (\%) }\end{array}$ & $\begin{array}{l}\text { Avg. T cell activation } \\
(\text { EC50, nM) }\end{array}$ & $\begin{array}{l}\text { Average max. T } \\
\text { cell activation (\%) }\end{array}$ \\
\hline 1 & 1 & 1.6 & 0.15 & 58 & 0.1 & 85 \\
\hline 2 & 12 & 1.1 & 0.76 & 58 & 0.7 & 82 \\
\hline 3 & 10 & 0.41 & 0.22 & 64 & 0.1 & 88 \\
\hline 4 & 12 & 0.95 & 0.70 & 57.1 & 0.3 & 85 \\
\hline 5 & 0 & NA & NA & NA & NA & NA \\
\hline 6 & 1 & 3.5 & 0.36 & 63 & 0.15 & 88 \\
\hline
\end{tabular}

Table 4. Comparison of Activity of BsAbs.

the mechanism of cytotoxicity, and to confirm T cell activation was not due to aggregation of BsAbs, leading to T cell crosslinking.

\section{Discussion}

The method described here combines three elements to allow for purification of BsAbs having the asymmetric "bipod" format. First, at least one v-region must be stable as a scFv. In general, this requires pre-screening for stability prior to formatting into a BsAb, since aggregated species will not be efficiently purified away from target species. Other moieties that lack a $\mathrm{CH} 1$ domain, such as a VHH or antigen fusion, would also lend themselves to this purification method. Second, this method depends on limiting the expression of Fab arm-containing species thereby "steering" the product towards scFv contaminants, which lack a CH1 domain. We found that a scFv-Fc to $\mathrm{HC}$ ratio of 2:1 resulted in over-expression of the $\mathrm{scFv}-\mathrm{Fc}$ chain in most cases. For those pairs having unusually high expressing $\mathrm{HC}$, or low expressing scFv, further increases in DNA ratio may be needed to achieve high purity. Third is the use of CH1-affinity chromatography to isolate highly pure target BsAb beyond what can be achieved by protein A capture alone. For the panels tested, application of all three of these elements could routinely achieve $95 \%$ purity.

This method was applied for expression at the $2 \mathrm{~mL}$ scale to allow for production of large panels of BsAbs, and due to practical limitations of $\mathrm{CH} 1$ affinity resin. One theoretical limitation of our method is the necessarily lower yields due to over-expression of one subunit However, in an HTP format, where only small amounts of product are needed for screening, this is not of primary concern. 
For single step purification, the imbalanced DNA ratio and $\mathrm{CH} 1$ purification steps result in highly pure target $\mathrm{BsAb}$, although a limitation is the necessarily lower yields due to over-expression of one subunit. An alternative approach is to introduce into the $\mathrm{CH} 1$-containing subunit mutations that disrupt protein $\mathrm{A}$ binding, such as those described previously ${ }^{20,21}$. BsAbs designed in this way allow purification specific to each chain - protein A for the scFv-Fc chain or $\mathrm{CH} 1$ for the HC + LC. This approach allows the DNA for each HC to be transfected at 1:1 molar ratio. Panels of proteins produced in this way would then be subjected to both protein A and CH1-based affinity purification to remove contaminants solely containing either $\mathrm{CH} 1$ or wild-type Fc.

Protein A-based affinity purification of Abs is generally preferred in scientific settings due to its high specificity, ease of capture and elution, availability of GMP-quality resin, and its ability to be continually recycled via sodium hydroxide regeneration. During development of this method, we noted that proteins captured directly from media supernatant onto $\mathrm{CH} 1$ resin could be efficiently eluted. However, the standard wash with $20 \mathrm{mM}$ sodium hydroxide resulted in retained endotoxin contamination in BsAbs purified using regenerated resin. Thus, column regeneration was performed with $40 \mathrm{mM} \mathrm{NaOH}$. Additionally, multiple purifications performed on regenerated columns showed successively lower yields. This may have been due to degradation of the $\mathrm{CH} 1$ affinity matrix or to accumulation of an unknown media component which led to lower binding capacity in subsequent purifications. For this reason, in large scale batches, we often perform a first step purification with protein A to remove the media supernatant component.

Large panels of BsAbs are needed to explore the complex design space around BsAbs that includes affinity, valency, epitope and format. The process described here is amenable to high-throughput production to support the optimization of BsAbs. In functional screening, BsAbs purified using our HTP method retained similar activity to the same BsAb purified at larger scale. These analyses will improve lead molecule selection for therapeutic programs.

\section{Methods}

Protein expression and purification. High-throughput. Antibody scFv-Fc, HC, and LC were cloned into single-gene plasmids each having an identical CMV promoter. Plasmids were transfected into ExpiCHO cells (ThermoFisher) at a ratio of 2:1:3 of scFv-Fc:HC:LC, according to the manufacturer's protocol. Cells were pelleted by centrifugation at $850 \times \mathrm{g}$ for 15 minutes and the culture supernatants were harvested. Plate based purification was performed in $2 \mathrm{~mL}$ 96-well $1 \mu \mathrm{m}$ glass filter plates prepared in-house. Supernatants were applied to either MabSelect SuRe LX resin (GE Healthcare) or CaptureSelect IgG-CH1 Affinity Matrix (Thermo Fisher), eluted according to the manufacturers' protocols and neutralized with Tris- $\mathrm{HCl}$.

Neutralized eluates $(0.5 \mathrm{~mL})$ containing purified BsAbs were dialyzed into PBS, pH 7.2 and filtered using a $1 \mathrm{~mL}$ 96-well $0.2 \mu \mathrm{m}$ Supor plate (Pall) prior to analysis. Quantification of each dialyzed elution pool was determined by measuring the absorbance at $280 \mathrm{~nm}$ using the Trinean DropSense (Unchained Labs). Purity of antibody solutions was assayed by analytical size-exclusion chromatography (SEC) using an Agilent AdvanceBio SEC. 300 A (Agilent) connected to an Agilent 1200 HPLC to monitor absorbance at 280 nanometers.

Large scale. Antibody scFv-Fc, HC, and LC were cloned into single-gene plasmids each having an identical CMV promoter. Plasmids were transfected into ExpiCHO cells (ThermoFisher) at a ratio of 2:1:3 of scFv-Fc: HC:LC, according to the manufacturer's protocol. Cells were pelleted by centrifugation at $4000 \times \mathrm{g}$ for $12 \mathrm{~min}-$ utes and the culture supernatants were harvested. Supernatants were applied to either mAbSelect Sure resin (GE Healthcare) or CaptureSelect CH1-XL affinity matrix (ThermoFisher) and eluted according to the manufacturers' protocols.

Eluates containing purified BsAbs were dialyzed into $1 \times \mathrm{PBS}$, pH 7.2 prior to analysis. Purity of antibody solutions was assayed by analytical size-exclusion chromatography (SEC) using a BioAssist G3SW $\mathrm{XL}_{\mathrm{C}}$ column (Tosoh). Capillary electrophoresis was performed using a Caliper LapChip GX II instrument according to the manufacturer's protocol. CH1-XL resin was regenerated by incubation with $40 \mathrm{mM} \mathrm{NaOH}$ for $30 \mathrm{~min}$.

Functional analysis. BsAbs were diluted to $40 \mathrm{nM}$ in assay media (RPMI $+10 \% \mathrm{FBS} \mathrm{HI}+1 \%$ Penicillin). Proteins were prepared in 3-fold serial dilutions for an 11-point titration in assay media in separate v-bottom polypropylene plates. CARNAVAL cells were diluted to $2 \times 10^{5}$ cells $/ \mathrm{ml}$ and plated at $20,000 \mathrm{cells} / \mathrm{well}$ in $100 \mathrm{~mL}$ of assay media. T cells were diluted to $2 \times 10^{6}$ cells $/ \mathrm{mL}$ and added to each well at $100,000 \mathrm{in} 50 \mathrm{~mL}$ of assay media to the assay plates containing tumor target cells. $50 \mathrm{uL}$ of serially diluted $\mathrm{BsAb}$ was added to the well starting from $10 \mathrm{nM}$ BsAb with 2-fold dilutions. Plates were incubated at $37^{\circ} \mathrm{C}, 5 \% \mathrm{CO} 2$ in a humidified cell culture incubator for 48 hours. After incubation, plates were centrifuged at $300 \times \mathrm{g}$ for $5 \mathrm{~min}$ to remove supernatants. Cells were washed by adding $150 \mathrm{uL}$ of staining buffer and spun again at $300 \times \mathrm{g}$ for $5 \mathrm{~min}$. Wash was repeated in the same manner by adding $200 \mathrm{uL}$ of staining buffer.

Staining solution mixture contained: APC-conjugated anti-Human CD4 (1:500) (Thermofisher cat. \# MHCD0405), APC-conjugated anti-Human CD8 (1:500) (Thermofisher cat. \# MHCD0805), and Brilliant Violet 421-conjugated anti-Human CD25 (1:500) (Biolegend cat. \# 302630). 50uL/well of staining solution mixture was added to the assay plates and incubated at room temperature for $20 \mathrm{~min}$ in the dark. 150uL staining buffer was added to all wells and wash twice by centrifugation at $400 \times \mathrm{g}$ for $3 \mathrm{~min}$ followed by removal of the supernatant. Vybrant DyeCycle Green (Thermofisher cat. \# V35004) was prepared at 1:25 k in Intellicyt running buffer. Cell pellets were resuspended in $20 \mathrm{uL}$ Intellicyt running buffer containing Sytox green live/dead stain (1:1000) or Vybrant DyeCycle Green, and plates were analyzed on the iQue PLUS Screener.

Gating strategy. A cell population was selected on FSC-H vs SSC-H, then T cell and tumor cell populations were selected on APC (RL1) vs SSC-H (Supplementary Fig. S3). Live and dead populations (live/dead stain) for both tumor and T cells from their respective dot plots on FSC-H vs Sytox Green (BL2) were selected. The live T 
cell population was used to gate activated T cell (CD25+) population on FSC-H vs Brilliant Violet (VL1). Gates were defined from control sample wells containing T-cells and tumor cells alone and in a mixture lacking BsAb. ForeCyt advanced metrics were used to calculate Tumor cell death as a percentage of dead Tumor cell events within tumor cell events, $\underline{\mathrm{T} \text { cell viability }}$ as a percentage of viable T cell events within $\mathrm{T}$ cells events, and Activated T cells as a percentage of Activated T cells events within viable T cells events.

Received: 6 January 2020; Accepted: 16 April 2020;

Published online: 05 May 2020

\section{References}

1. Labrijn, A. F., Janmaat, M. L., Reichert, J. M. \& Parren, P. Bispecific antibodies: a mechanistic review of the pipeline. Nat Rev Drug Discov 18, 585-608, https://doi.org/10.1038/s41573-019-0028-1 (2019).

2. Andreev, J. et al. Bispecific Antibodies and Antibody-Drug Conjugates (ADCs) Bridging HER2 and Prolactin Receptor Improve Efficacy of HER2 ADCs. Mol Cancer Ther 16, 681-693, https://doi.org/10.1158/1535-7163.MCT-16-0658 (2017).

3. Goulet, D. R. \& Atkins, W. M. Considerations for the Design of Antibody-Based Therapeutics. J Pharm Sci, https://doi.org/10.1016/j. xphs.2019.05.031 (2019).

4. Zhu, Y. et al. Identification of CD112R as a novel checkpoint for human T cells. J Exp Med 213, 167-176, https://doi.org/10.1084/ jem.20150785 (2016)

5. Yu, S. et al. Recent advances of bispecific antibodies in solid tumors. J Hematol Oncol 10, 155, https://doi.org/10.1186/s13045-0170522-z (2017).

6. Brinkmann, U. \& Kontermann, R. E. The making of bispecific antibodies. MAbs 9, 182-212, https://doi.org/10.1080/19420862.201 6.1268307 (2017).

7. Saunders, K. O. Conceptual Approaches to Modulating Antibody Effector Functions and Circulation Half-Life. Front Immunol 10, 1296, https://doi.org/10.3389/fimmu.2019.01296 (2019).

8. Wang, X., Mathieu, M. \& Brezski, R. J. IgG Fc engineering to modulate antibody effector functions. Protein Cell 9, 63-73, https://doi. org/10.1007/s13238-017-0473-8 (2018).

9. Ha, J. H., Kim, J. E. \& Kim, Y. S. Immunoglobulin Fc Heterodimer Platform Technology: From Design to Applications in Therapeutic Antibodies and Proteins. Front Immunol 7, 394, https://doi.org/10.3389/fimmu.2016.00394 (2016).

10. Labrijn, A. F. et al. Efficient generation of stable bispecific IgG1 by controlled Fab-arm exchange. Proc Natl Acad Sci USA 110, 5145-5150, https://doi.org/10.1073/pnas.1220145110 (2013).

11. Von Kreudenstein, T. S. et al. Improving biophysical properties of a bispecific antibody scaffold to aid developability: quality by molecular design. MAbs 5, 646-654, https://doi.org/10.4161/mabs.25632 (2013).

12. Lewis, S. M. et al. Generation of bispecific IgG antibodies by structure-based design of an orthogonal Fab interface. Nat Biotechnol 32, 191-198, https://doi.org/10.1038/nbt.2797 (2014).

13. Dillon, M. et al. Efficient production of bispecific IgG of different isotypes and species of origin in single mammalian cells. MAbs 9 , 213-230, https://doi.org/10.1080/19420862.2016.1267089 (2017).

14. Liu, Z. et al. A novel antibody engineering strategy for making monovalent bispecific heterodimeric IgG antibodies by electrostatic steering mechanism. J Biol Chem 290, 7535-7562, https://doi.org/10.1074/jbc.M114.620260 (2015).

15. De Nardis, C. et al. A new approach for generating bispecific antibodies based on a common light chain format and the stable architecture of human immunoglobulin G1. J Biol Chem 292, 14706-14717, https://doi.org/10.1074/jbc.M117.793497 (2017).

16. Wang, Q. et al. Design and Production of Bispecific Antibodies. Antibodies (Basel) 8, https://doi.org/10.3390/antib8030043 (2019).

17. Spreter Von Kreudenstein, T., Lario, P. I. \& Dixit, S. B. Protein engineering and the use of molecular modeling and simulation: the case of heterodimeric Fc engineering. Methods 65, 77-94, https://doi.org/10.1016/j.ymeth.2013.10.016 (2014).

18. Mains, P. E. \& Sibley, C. H. The requirement of light chain for the surface deposition of the heavy chain of immunoglobulin M. J Biol Chem 258, 5027-5033 (1983).

19. Grugan, K. D. et al. Fc-mediated activity of EGFR $\times$ c-Met bispecific antibody JNJ-61186372 enhanced killing of lung cancer cells. MAbs 9, 114-126, https://doi.org/10.1080/19420862.2016.1249079 (2017).

20. Tustian, A. D., Endicott, C., Adams, B., Mattila, J. \& Bak, H. Development of purification processes for fully human bispecific antibodies based upon modification of protein A binding avidity. MAbs 8, 828-838, https://doi.org/10.1080/19420862.2016.116019 2 (2016).

21. Zwolak, A. et al. Rapid Purification of Human Bispecific Antibodies via Selective Modulation of Protein A Binding. Sci Rep 7, 15521, https://doi.org/10.1038/s41598-017-15748-0 (2017).

\section{Acknowledgements}

We thank David Poon (Zymeworks) and Peter Haytko (Janssen R\&D) for helpful discussions regarding heterodimerization mutants and purification strategies.

\section{Author contributions}

A.Z. and T.N. designed molecules. M.D., K.K., K.B., C.S., J.S. and H.H. purified proteins and analyzed data. H.J., S.L.L.P., R.G., and S.S. designed experiments. N.M. and G.P. performed functional analysis. A.Z. and T.N. conceived of the experiments, designed molecules, and A.Z., T.N., K.K., N.M., and R.G. wrote the manuscript.

\section{Competing interests}

All authors are full-time employees of Janssen Pharmaceuticals, Ltd. Janssen has disclosed a partnership with Zymeworks.

\section{Additional information}

Supplementary information is available for this paper at https://doi.org/10.1038/s41598-020-64536-w.

Correspondence and requests for materials should be addressed to A.Z.

Reprints and permissions information is available at www.nature.com/reprints.

Publisher's note Springer Nature remains neutral with regard to jurisdictional claims in published maps and institutional affiliations. 
(c) (i) Open Access This article is licensed under a Creative Commons Attribution 4.0 International License, which permits use, sharing, adaptation, distribution and reproduction in any medium or format, as long as you give appropriate credit to the original author(s) and the source, provide a link to the Creative Commons license, and indicate if changes were made. The images or other third party material in this article are included in the article's Creative Commons license, unless indicated otherwise in a credit line to the material. If material is not included in the article's Creative Commons license and your intended use is not permitted by statutory regulation or exceeds the permitted use, you will need to obtain permission directly from the copyright holder. To view a copy of this license, visit http://creativecommons.org/licenses/by/4.0/.

(C) The Author(s) 2020 